\title{
Risk Factors and Blood Pressure Control Targets Associated With the Clinical Outcome of Elderly COVID-19 Patients With Hypertension: a Retrospective Cohort Study
}

\section{Ting Li}

Chinese PLA General Hospital https://orcid.org/0000-0002-3550-7034

\section{Maomao Wang}

Second Military Medical University

\section{Yifei Wang}

Chinese PLA General Hospital

\section{Pei Zhang}

Chinese PLA General Hospital

\section{Yang Wang}

Chinese PLA General Hospital

\section{Jing Zeng}

Chinese PLA General Hospital

\section{Laixing Wang}

The Second Military Medical University

\section{Chunlin Li}

Chinese PLA General Hospital

\section{Yanping Gong ( $\sim$ gypmrp@163.com )}

Chinese PLA General Hospital

\section{Research}

Keywords: COVID-19, Hypertension, Outcome, Risk factors, Cut-off values

Posted Date: July 15th, 2021

DOl: https://doi.org/10.21203/rs.3.rs-711780/v1

License: (1) This work is licensed under a Creative Commons Attribution 4.0 International License. Read Full License 


\section{Abstract}

Background: COVID-19 is a global pandemic, especially among the elderly. Our study aimed to explore the risk factors and identify the blood pressure control targets associated with the clinical outcome of elderly COVID-19 patients with hypertension.

Methods: In this retrospective cohort study, elderly COVID-19 patients who were admitted to Wuhan Huoshenshan Hospital from February 8 to 17, 2020 was included. Demographic, medical history, clinical data, and laboratory test data were collected from medical records. The adverse clinical outcomes were intensive care unit (ICU) admission and death. Difference between hypertension and non-hypertension groups were compared. Hypertension group were further divided into 3 subgroups according to their maximum blood pressures. Kaplan-Meier ( $\mathrm{K}-\mathrm{M})$ method was used to find the differences both between hyperntesion and non-hypertension groups, and among the 3 hypertension subgroups. Univariable and multivariable Cox proportional hazards regression model were used to find risk factors.

Results: All 133 elderly COVID-19 patients (79 patients with hypertension) were included. (1) Univariate analysis between hypertension and non-hypertension patients showed most laboratory tests were significantly $(P<0.05$, or $P<0.01)$, particularly in adverse clinical outcomes $(32.91 \%$ vs $7.41 \%$ at 30 days, $P<0.05)$. (2) Multivariate Cox proportional hazards models confirmed hypertension (HR 3.202, 95\% Cl:1.164 - 8.807) were the most important independent risk factors of outcomes in elderly patients, as well as low lymphocyte count, while the statistical difference of other values diminished. (3) Hypertension group were further divided into 3 subgroups according to their maximum blood pressures. $\mathrm{K}-\mathrm{M}$ analysis showed maximum systolic blood pressure (SBP) $\geq 160 \mathrm{mmHg}$ subgroup $(P<0.01)$ and maximum blood pressure (DBP) $\geq 90 \mathrm{mmHg}$ subgroup $(P<0.05)$ experienced more adverse outcomes than others. (4) Multivariate Cox-proportional hazard model confirmed that maximum SBP $\geq 160 \mathrm{mmHg}$ and maximum DBP $\geq 90 \mathrm{mmHg}$ were risk factors (HR 8.279, 95\% Cl: 1.346, 50.914; HR 5.080, 95\% Cl: 1.606,16.071; respectively).

Conclusions: Hypertension is the most important independent risk factor of adverse outcomes in elderly COVID-19 patients, controlling the maximum blood pressure levels under 160/90 $\mathrm{mmHg}$ will decrease large part risks of adverse outcomes, the first week are key treatment period for patient prognosis.

\section{Background}

Coronavirus disease-2019 (COVID-19) is a life-threatening infection caused by the severe acute respiratory syndrome coronavirus-2 (SARS-CoV-2) virus(1). It has caused billions infections and millions deaths $(2,3)$. Based on the global pandemic of COVID-19, it has become critical to study the risk factors of clinical prognosis.

It has been previously reported that patients with older age and chronic underlying conditions might have a high risk of experiencing intensive care and death. Moreover, current clinical data suggest that, in COVID-19 patients, hypertension can worsen outcomes and increase the risk of admission to intensive 
care unit (ICU) (4). Previous clinical trials showed that SARSCoV-2 enters cells through angiotensinconverting enzyme 2(ACE2) receptors(5); high blood pressure is likely to increase COVID-19 mortality by affecting lung function and impairing oxygen delivery(6). As the COVID-19 pandemic progresses, the high incidence of hypertension makes this problem particularly worrying. However, it is not clear whether the mortality of COVID-19 patients with hypertension is related with the level of blood pressure control(7); there is a lack of detailed prognosis studies of hypertension at different blood pressure levels; even not to say few studies have explicitly identified blood pressure control cut-off points in hypertension comorbidities(8).

Thus, in this study, we aim to report the correlation between hypertension and COVID-19 adverse outcomes in elderly hypertensive population, and try to found the risk factors and the control cut-off value for elderly hypertension population.

\section{Methods}

\section{Study design and participants}

This single-center, retrospective cohort study was conducted at the Huoshenshan Hospital in Wuhan, which was approved by the Institutional Ethics Committee of Huoshenshan Hospital (HSSLL030). Considering the urgency of the COVID-19 pandemic, the need for informed consent forms were waived by the ethics board of the hospitals. All participants included in the study were diagnosed with COVID-19 who were hospitalized at Huoshenshan Hospital from February 8 to 17, 2020. According to the World Health Organization's temporary guidelines, COVID-19 was diagnosed based on SARS-CoV-2 nucleic-acid swab test and/or chest computed tomography (CT) scanning (the criteria of the New Coronavirus Pneumonia Prevention and Control Program, sixth edition, National Health Commission of China, 2020(9)).

\section{Data collection}

By reviewing electronic medical records, we obtained medical history, demographic data, signs and symptoms, laboratory tests, and chest CT scans. The outcomes were followed up until March 29, 2020. The adverse clinical outcomes were intensive care unit (ICU) admission and death. The clinical characteristics, laboratory tests, incidence of complications were monitored. All data were checked by another researcher to ascertain their accuracy and were analysed by an integrated research team which includes physicians and statisticians.

\section{Definitions}

All patients with COVID-19 met the following criteria: (1) fever and/or other typical respiratory symptoms, (2) typical CT image manifestations of viral pneumonia, and (3) positive SARS-COV-2 RNA by reverse 
transcription-polymerase chain reaction (RT-PCR) and/or gene diagnosis. And severe COVID-19 patients met the following criteria: (1) respiratory distress, with respiratory rate $\geq 30$ beats/min, (2) finger oxygen saturation $\leq 93 \%$ at rest, and (3) ratio of the partial pressure of arterial oxygen to the fraction of inspired oxygen $(\mathrm{PaO} 2: \mathrm{FiO} 2) \leq 300 \mathrm{mmHg}$.

Hypertension was designated based on the patient's medical history and guideline for diagnosis according to their blood pressure level at admission(higher than $140 / 90 \mathrm{mmHg}$ ). Patients with transient hormone-induced hypertension in the ICU were not included in the hypertension group. Blood pressure of patient were all measured and recorded several times depending on the patient's condition, the classification of hypertension made depending the 2020 International Society of Hypertension Global Hypertension Practice Guidelines(10). Maximum blood pressure, minimum blood pressure, pulse pressure and mean arterial pressure were all extracted based from the above data. Diabetes, cardiac injury, acute liver injury, and acute kidney injury status were designated based on the patient's medical history and guideline for diagnosis(11).

\section{Statistical analysis}

Our statistical analyses were conducted with SPSS (version 26.0) and GraphPad Prism (version 8.0) software. We made no assumptions regarding missing data. Categorical variables are described as frequency rates and percentage (\%). Continuous variables are described as mean \pm standard deviation (SD) or median (interquartile range (IQRs)). We used the Student's t-tests (normally distributed) or Mann Whitney test (non-normally distributed) for continuous variables between two groups, and used the Fisher's exact test or $\chi^{2}$ test for categorical variables between two groups. To explore the risk factors associate with adverse clinical outcomes, univariable and multivariable Cox proportional hazards regression model was performed. All the variables included in the final models were based on clinical and scientific understanding, previous findings, and the results of univariable analyses. We divided the hypertension group into different blood pressure subgroups according to the latest guideline and analyzed the composition ratio of each subgroup. Kaplan-Meier $(\mathrm{K}-\mathrm{M})$ method was further used to find the best blood pressure control level in the COVID-19 hypertension group. A two-sided $P$ value, 0.05 was considered statistically significant.

\section{Results}

\section{Population}

A total of 180 patients were initially screened for the study, and 47 patients younger than 60 years old were excluded. According to the clinical diagnosis and/or medical history on admission, the 133 patients were divided into two groups: hypertension patients $(n=79)$ and non-hypertension patients $(n=54)$ (Fig. 1). A total of $36(27.1 \%)$ patients were defined as adverse clinical outcomes, including ICU admission $(n=34,25.6 \%)$ and in-hospital death $(n=16,12.0 \%$, of which 14 had overlapping with ICU patients). 
The difference of general conditions between hypertension and non-hypertension patients

The median age was 68 years (IQR, 64-73 years) and 68 cases (51.1\%) were male in this study (Table 1). Hypertension $(n=79,59.4 \%)$, diabetes $(n=57,42.9 \%)$, and coronary atherosclerotic heart disease $(n=18$, $13.5 \%)$ were the three most common comorbidities. Cough $(n=114,85.7 \%)$, fever $(n=109,82.0 \%)$, and shortness of breath $(n=88,66.2 \%)$ were the three most common symptoms (Table 1$)$. 
Table 1

Demographics and symptomatic characteristics of patients with COVID-19

\begin{tabular}{|c|c|c|c|c|}
\hline & \multirow{2}{*}{$\begin{array}{l}\text { Total } \\
(n=133)\end{array}$} & \multirow{2}{*}{$\begin{array}{l}\text { Hypertension } \\
(\mathrm{n}=79)\end{array}$} & \multirow{2}{*}{$\begin{array}{l}\text { Non-hypertension } \\
(n=54)\end{array}$} & \multirow[t]{2}{*}{$P$ value } \\
\hline & & & & \\
\hline Age, median (IQR) & $68(64-73)$ & $69(64-75)$ & $67(63-71)$ & 0.122 \\
\hline Gender & & & & 0.221 \\
\hline Male & $68(51.1 \%)$ & $44(55.9 \%)$ & $24(44.4 \%)$ & \\
\hline Female & $65(48.9 \%)$ & $35(44.3 \%)$ & $30(55.6 \%)$ & \\
\hline Smoking history & $9(7.1 \%)$ & $8(10.8 \%)$ & $1(1.9 \%)$ & 0.062 \\
\hline Cluster disease & $11(8.3 \%)$ & $7(8.9 \%)$ & $4(7.4 \%)$ & 0.765 \\
\hline Diagnosis method & & & & 0.119 \\
\hline RT-PCR & $114(85.7 \%)$ & 65(82.3\%) & $49(90.6 \%)$ & \\
\hline Gene & $1(0.8 \%)$ & $0(0.0 \%)$ & $1(1.9 \%)$ & \\
\hline Clinical diagnosis & $18(13.5 \%)$ & $14(17.7 \%)$ & $4(7.4 \%)$ & \\
\hline \multicolumn{5}{|l|}{ Comorbidities } \\
\hline Hypertension & $79(59.4 \%)$ & $79(100.0 \%)$ & $0(0.0 \%)$ & / \\
\hline Diabetes & $57(42.9 \%)$ & $41(51.9 \%)$ & $16(29.6 \%)$ & 0.013 \\
\hline $\mathrm{CHD}$ & $18(13.5 \%)$ & $13(16.5 \%)$ & $5(9.3 \%)$ & 0.233 \\
\hline Cerebrovascular disease & $10(7.5 \%)$ & $8(10.1 \%)$ & $2(3.7 \%)$ & 0.168 \\
\hline Liver disease & $3(2.3 \%)$ & $2(2.5 \%)$ & $1(1.9 \%)$ & 0.795 \\
\hline Kidney disease & $5(3.8 \%)$ & $4(5.1 \%)$ & $0(0.0 \%)$ & 0.407 \\
\hline COPD & $4(3.0 \%)$ & $2(2.5 \%)$ & $2(3.7 \%)$ & 0.698 \\
\hline Malignancy & $7(5.3 \%)$ & $4(5.1 \%)$ & $3(5.6 \%)$ & 0.901 \\
\hline \multicolumn{5}{|l|}{ Signs and symptoms } \\
\hline Fever & $109(82.0 \%)$ & $62(78.5 \%)$ & $47(87.0 \%)$ & 0.255 \\
\hline Cough & 114(85.7\%) & $70(88.6 \%)$ & $44(81.5 \%)$ & 0.249 \\
\hline Expectoration & $23(17.3 \%)$ & $19(24.1 \%)$ & $4(7.4 \%)$ & 0.013 \\
\hline Rhinorrhoea & $3(2.3 \%)$ & $1(1.3 \%)$ & $2(3.7 \%)$ & 0.325 \\
\hline Haemoptysis & $1(0.8 \%)$ & $1(1.3 \%)$ & $0(0.0 \%)$ & 0.407 \\
\hline Shortness of breath & $88(66.2 \%)$ & $54(68.4 \%)$ & $34(63.0 \%)$ & 0.519 \\
\hline
\end{tabular}




\begin{tabular}{|c|c|c|c|c|}
\hline & Total & Hypertension & Non-hypertension & $P$ value \\
\hline & $(n=133)$ & $(n=79)$ & $(n=54)$ & \\
\hline Fatigue & $86(64.7 \%)$ & $46(58.2 \%)$ & $40(74.1 \%)$ & 0.060 \\
\hline Myalgia & $52(39.1 \%)$ & $24(30.4 \%)$ & $28(51.9 \%)$ & 0.013 \\
\hline Diarrhoea & $13(9.8 \%)$ & $9(11.4 \%)$ & $4(7.4 \%)$ & 0.447 \\
\hline Nausea and vomiting & $12(9.0 \%)$ & $8(10.1 \%)$ & $4(7.4 \%)$ & 0.447 \\
\hline Arrhythmias & $8(6.0 \%)$ & $6(7.6 \%)$ & $2(3.7 \%)$ & 0.354 \\
\hline Palpitation & $4(3.0 \%)$ & $2(2.5 \%)$ & $2(3.7 \%)$ & 0.698 \\
\hline Dizziness & $5(3.8 \%)$ & $4(5.1 \%)$ & $1(1.9 \%)$ & 0.339 \\
\hline Headache & $4(3.0 \%)$ & $1(1.3 \%)$ & $3(5.6 \%)$ & 0.155 \\
\hline
\end{tabular}

There was no difference in age, gender, smoking history and diagnostic methods between hypertension and non-hypertension patients $(P>0.05)$. Among all comorbidities, only diabetes had a statistically significant difference between hypertension and non-hypertension patients $(P<0.01)$. Of all the symptoms recorded, only expectoration and myalgia had statistically significant differences between hypertension and non-hypertension patients $(P<0.01)($ Table 1$)$.

\section{The difference of clinical laboratory between hypertension and non-hypertension patients}

Except c-reactive protein (CRP) and D-dimer, mean levels of other indices of the 133 patients were in normal range (Table 2). All BG indices, kidney function (urea nitrogen, BUN), nutrition (albumin), myocardial enzyme (creatine kinase-MB, lactate dehydrogenase), inflammatory indices (CRP, white blood cell count, neutrophil percentage, neutrophil count, lymphocyte percentage), hemoglobin, coagulation indices (prothrombin time, PT) were significantly higher in hypertension patients than non-hypertension patients $(P<0.05)$. Other blood routine examination (lymphocyte count et al.) and biochemical detection (liver function et al.) in the hypertension group were inferior to the non-hypertension group $(P<0.05)$ (Table 2). 
Table 2

Laboratory parameters of patients with COVID-19

$\begin{array}{lllll}\begin{array}{l}\text { Reference } \\ \text { values }\end{array} & \begin{array}{l}\text { Total } \\ (n=133)\end{array} & \begin{array}{l}\text { Hypertension } \\ (n=79)\end{array} & \begin{array}{l}\text { Non- } \\ \text { hypertension } \\ (n=54)\end{array} & \begin{array}{l}P \\ \text { value }\end{array} \\ & & & \end{array}$

\section{Blood glucose}

\begin{tabular}{|c|c|c|c|c|}
\hline $\begin{array}{l}\text { Maximum BG, median } \\
\text { (IQR), } \mathrm{mmol} / \mathrm{L}\end{array}$ & $3.9-6.11$ & $\begin{array}{l}5.61(4.93- \\
14.40)\end{array}$ & $\begin{array}{l}9.06(5.06- \\
17.20)\end{array}$ & $\begin{array}{l}5.16(4.80- \\
7.28)\end{array}$ \\
\hline $\begin{array}{l}\text { Average BG at the day } \\
\text { of admission, median }\end{array}$ & $3.9-6.11$ & $\begin{array}{l}5.38(4.80- \\
8.86)\end{array}$ & $\begin{array}{l}7.07(4.84- \\
11.04)\end{array}$ & $\begin{array}{l}5.06(4.71- \\
7.28)\end{array}$ \\
\hline
\end{tabular}

\section{Biochemical detection}

\begin{tabular}{|c|c|c|c|c|c|}
\hline $\begin{array}{l}\text { Total protein, median } \\
(\text { IQR), g/L }\end{array}$ & $65-85$ & $\begin{array}{l}61.10(57.35- \\
65.65)\end{array}$ & $\begin{array}{l}60.90(57.20- \\
63.90)\end{array}$ & $\begin{array}{l}61.40(58.35- \\
67.15)\end{array}$ & 0.245 \\
\hline ALT, median (IQR), IU/L & $7-40$ & $\begin{array}{l}23.95(16.55- \\
41.00)\end{array}$ & $\begin{array}{l}23.90(17.40- \\
41.00)\end{array}$ & $\begin{array}{l}24.20(15.40- \\
41.05)\end{array}$ & 0.753 \\
\hline AST, median (IQR), IU/L & $7-45$ & $\begin{array}{l}23.30(16.75- \\
34.15)\end{array}$ & $\begin{array}{l}24.60(18.40- \\
37.05)\end{array}$ & $\begin{array}{l}21.90(15.25- \\
28.20)\end{array}$ & 0.070 \\
\hline $\begin{array}{l}\text { Albumin, median (IQR), } \\
\mathrm{g} / \mathrm{L}\end{array}$ & $40-55$ & $\begin{array}{l}33.40(30.30- \\
36.60)\end{array}$ & $\begin{array}{l}32.90(29.40- \\
36.30)\end{array}$ & $\begin{array}{l}34.15(30.96- \\
37.55)\end{array}$ & 0.042 \\
\hline $\begin{array}{l}\text { BUN, median (IQR), } \\
\mathrm{mmol} / \mathrm{L}\end{array}$ & $2.6-7.5$ & $\begin{array}{l}5.12(4.05- \\
6.85)\end{array}$ & $\begin{array}{l}5.75(4.33- \\
8.34)\end{array}$ & $\begin{array}{l}4.60(3.82- \\
5.62)\end{array}$ & $\begin{array}{l}\square \\
0.001\end{array}$ \\
\hline $\begin{array}{l}\text { Creatinine, median } \\
(\mathrm{IQR}), \mu \mathrm{mol} / \mathrm{L}\end{array}$ & $41-73$ & $\begin{array}{l}\text { 67.45(56.78- } \\
80.75)\end{array}$ & $\begin{array}{l}\text { 68.90(58.13- } \\
83.53)\end{array}$ & $\begin{array}{l}\text { 64.15(54.05- } \\
74.28)\end{array}$ & 0.080 \\
\hline $\begin{array}{l}\text { Uric acid, median (IQR), } \\
\mu \mathrm{mol} / \mathrm{L}\end{array}$ & $142-340$ & $\begin{array}{l}258.00(190.50- \\
329.00)\end{array}$ & $\begin{array}{l}273.00(180.00- \\
341.00)\end{array}$ & $\begin{array}{l}233.00(199.00- \\
303.50)\end{array}$ & 0.410 \\
\hline $\begin{array}{l}\text { Total bile acid, median } \\
\text { (IQR), } \mu \mathrm{mol} / \mathrm{L}\end{array}$ & $0-10$ & $\begin{array}{l}3.75(2.53- \\
5.60)\end{array}$ & $\begin{array}{l}4.30(2.50- \\
6.05)\end{array}$ & $\begin{array}{l}3.50(2.70- \\
5.50)\end{array}$ & 0.331 \\
\hline $\begin{array}{l}\text { Creatine kinase, } \\
\text { median (IQR), IU/L }\end{array}$ & $24-170$ & $\begin{array}{l}43.20(29.15- \\
72.80)\end{array}$ & $\begin{array}{l}44.60(31.30- \\
74.70)\end{array}$ & $\begin{array}{l}40.60(26.60- \\
65.80)\end{array}$ & 0.171 \\
\hline $\begin{array}{l}\text { Creatine kinase-MB, } \\
\text { median (IQR), IU/L }\end{array}$ & $0-24$ & $\begin{array}{l}10.00(7.05- \\
12.95)\end{array}$ & $\begin{array}{l}10.80(8.15- \\
14.25)\end{array}$ & $\begin{array}{l}7.95(6.13- \\
11.65)\end{array}$ & $\begin{array}{l}\square \\
0.001\end{array}$ \\
\hline $\begin{array}{l}\text { LDH, median (IQR), } \\
\text { IU/L }\end{array}$ & $120-250$ & $\begin{array}{l}212.80(173.93- \\
326.55)\end{array}$ & $\begin{array}{l}257.80(185.05- \\
382.50)\end{array}$ & $\begin{array}{l}190.70(167.15- \\
250.45)\end{array}$ & $\begin{array}{l}0 \\
0.001\end{array}$ \\
\hline \multicolumn{6}{|l|}{ Inflammatory indices } \\
\hline $\begin{array}{l}\text { CRP, median (IQR), } \\
\mathrm{mg} / \mathrm{L}\end{array}$ & $0-4$ & $\begin{array}{l}8.97(2.41- \\
53.58)\end{array}$ & $\begin{array}{l}16.03(2.71- \\
81.70)\end{array}$ & $\begin{array}{l}6.37(1.96- \\
21.00)\end{array}$ & 0.021 \\
\hline
\end{tabular}




\begin{tabular}{|c|c|c|c|c|c|}
\hline $\begin{array}{l}\text { White blood cell count, } \\
\text { median (IQR), } \times 10^{9} / \mathrm{L}\end{array}$ & $3.5-9.5$ & $\begin{array}{l}6.35(4.70- \\
8.35)\end{array}$ & $\begin{array}{l}6.90(5.20- \\
9.00)\end{array}$ & $\begin{array}{l}5.70(4.15- \\
7.25)\end{array}$ & 0.011 \\
\hline $\begin{array}{l}\text { Neutrophil percentage, } \\
\text { mean } \pm \text { SD, \% }\end{array}$ & $40-75$ & $70.15 \pm 14.67$ & $70.97 \pm 15.05$ & $65.95 \pm 12.98$ & 0.021 \\
\hline $\begin{array}{l}\text { Lymphocyte } \\
\text { percentage, mean } \pm \text { SD, } \\
\%\end{array}$ & $20-50$ & $20.85 \pm 12.02$ & $19.90 \pm 11.91$ & $23.99 \pm 11.78$ & 0.023 \\
\hline $\begin{array}{l}\text { Neutrophil count, } \\
\text { median (IQR), } \times 10^{9} / \mathrm{L}\end{array}$ & $1.8-6.3$ & $\begin{array}{l}4.55(2.85- \\
6.77)\end{array}$ & $\begin{array}{l}5.15(3.30- \\
7.21)\end{array}$ & $\begin{array}{l}3.63(2.32- \\
5.45)\end{array}$ & $\begin{array}{l}\square \\
0.001\end{array}$ \\
\hline $\begin{array}{l}\text { Lymphocyte count, } \\
\text { median (IQR), } \times 10^{9} / \mathrm{L}\end{array}$ & $1.1-3.2$ & $\begin{array}{l}1.18(0.75- \\
1.64)\end{array}$ & $\begin{array}{l}1.08(0.65- \\
1.60)\end{array}$ & $\begin{array}{l}1.24(0.94- \\
1.67)\end{array}$ & 0.170 \\
\hline $\begin{array}{l}\text { Monocyte count, mean } \\
\pm \mathrm{SD}, \times 10^{9} / \mathrm{L}\end{array}$ & $0.1-0.6$ & $0.47 \pm 0.23$ & $0.47 \pm 0.20$ & $0.47 \pm 0.20$ & 0.920 \\
\hline $\begin{array}{l}\text { Eosinophil count, } \\
\text { median (IQR), } \times 10^{9} / \mathrm{L}\end{array}$ & $\begin{array}{l}0.02- \\
0.52\end{array}$ & $\begin{array}{l}0.06(0.03- \\
0.12)\end{array}$ & $\begin{array}{l}0.05(0.02- \\
0.12)\end{array}$ & $\begin{array}{l}0.06(0.03- \\
0.11)\end{array}$ & 0.689 \\
\hline $\begin{array}{l}\text { Hemoglobin, mean } \pm \\
\text { SD, g/L }\end{array}$ & $130-175$ & $121.59 \pm 17.75$ & $125.19 \pm 18.49$ & $120.03 \pm 12.95$ & 0.025 \\
\hline $\begin{array}{l}\text { Platelet count, mean } \pm \\
\mathrm{SD}, \times 10^{9} / \mathrm{L}\end{array}$ & $125-350$ & $242.02 \pm 94.16$ & $228.30 \pm 92.87$ & $242.39 \pm 93.06$ & 0.195 \\
\hline \multicolumn{6}{|l|}{ Coagulation indices } \\
\hline $\begin{array}{l}\text { PT, median (IQR), } \\
\text { seconds }\end{array}$ & $9.2-15$ & $\begin{array}{l}13.32(12.44- \\
14.38)\end{array}$ & $\begin{array}{l}13.73(12.61- \\
14.99)\end{array}$ & $\begin{array}{l}13.12(12.10- \\
13.85)\end{array}$ & 0.012 \\
\hline $\begin{array}{l}\text { APPT, median (IQR), } \\
\text { seconds }\end{array}$ & $21-37$ & $\begin{array}{l}27.96(25.60- \\
30.19)\end{array}$ & $\begin{array}{l}28.15(25.59- \\
30.15)\end{array}$ & $\begin{array}{l}27.65(25.65- \\
30.38)\end{array}$ & 0.586 \\
\hline $\begin{array}{l}\text { D-dimer, median (IQR), } \\
\mathrm{mg} / \mathrm{L}\end{array}$ & $0-0.55$ & $\begin{array}{l}0.78(0.45- \\
2.90)\end{array}$ & $\begin{array}{l}0.82(0.50- \\
2.76)\end{array}$ & $\begin{array}{l}0.70(0.37- \\
2.91)\end{array}$ & 0.384 \\
\hline \multicolumn{6}{|c|}{$\begin{array}{l}\text { Abbreviation: IQR, interquartile range; ALT, alanine aminotransferase; AST, aspartate } \\
\text { aminotransferase; BUN, urea nitrogen; LDH, lactate dehydrogenase; CRP, C-reactive protein; PT, } \\
\text { prothrombin time; APPT, activated partial thromboplastin time }\end{array}$} \\
\hline
\end{tabular}

\section{The difference of adverse clinical outcomes between hypertension and non-hypertension patients}

There were $49.4 \%$ hypertension patients with mild to moderate symptoms and $50.6 \%$ with severe symptoms, compared to the $88.9 \%$ non-hypertension patients with mild to moderate symptoms and $11.1 \%$ with severe symptoms (Fig. 2A a-b). K-M analysis were performed to calculate the percent of clinical outcomes between hypertension group and non-hypertension group. At the last follow-up visit, 31 patients in the hypertension group (39.2\%, in which $24.0 \%$ of on death in ICU and $15.2 \%$ of death) and 5 in the non-hypertension group ( $9.3 \%$, in which $1.9 \%$ of on death in ICU, $7.4 \%$ of death) had adverse clinical 
outcomes (Fig. 2A c-d), and the overall clinical outcomes rates in the two groups differed significantly on the basis of the stratified log-rank test. It showed 32.91\% (at 30 days) and 39.24\% (at 60 days) adverse clinical outcomes in hypertension patients, compared to the $7.41 \%$ (at 30 days) and $9.26 \%$ (at 60 days) in non-hypertension patients. The adverse event in hypertension patients began to increase sharply at about 5 days and relaxed after 30 days. And it reached the platform at about 45 days (Fig. 2B).

\section{Risk factors of adverse clinical outcomes in elderly COVID-19 patients}

Univariate Cox hazard analysis was used to screen for statistically significant variables associated with adverse clinical outcomes. In the total population, hypertension (HR 4.937) and diabetes (HR 5.821) were the most significantly two risk factors related with adverse clinical outcomes. Average BG, BUN, neutrophil percentage, neutrophil count, maximum BG, white blood cell count, PT were the following 8 risk factors with HR range from 1.085-1.154 (Table 3). The Cox hazard analysis also showed that the top 10 risk factors of univariate Cox analysis in hypertension group were the same with that of total population only different in rank order (Table 3). Multivariate Cox-proportional hazard model analysis was conducted to further certify whether above variables got from univariate Cox affected patient clinical outcomes independently. The results demonstrated hypertension was the most important independent factor that relates with higher adverse clinical outcomes (HR 3.202, 95\% Cl:1.164-8.807; Fig. 3); and other significantly risk factors include low lymphocyte count, hemoglobin, CRP, LDH and platelet count.

Table 3. Univariate Cox hazard analysis to further certify whether variables affected patient clinical outcomes (Only if $\mathrm{P}<0.05$ is listed in the table) 


\begin{tabular}{|c|c|c|c|c|c|c|c|c|c|}
\hline \multirow[t]{2}{*}{ Factors } & \multicolumn{3}{|c|}{ Total $(\mathrm{N}=133)$} & \multicolumn{3}{|c|}{ Hypertension $(N=79)$} & \multicolumn{3}{|c|}{ Non-hypertension $(\mathrm{N}=54)$} \\
\hline & Rank & $\mathrm{HR}$ & $P$ value & Rank & $\mathrm{HR}$ & $P$ value & Rank & $\mathrm{HR}$ & $P$ value \\
\hline Age & 11 & 1.051 & 0.017 & l & $l$ & l & 8 & 1.180 & 0.024 \\
\hline Hypertension & 2 & 4.937 & 0.001 & l & $l$ & l & $l$ & l & l \\
\hline Diabetes & 1 & 5.821 & $<0.001$ & 1 & 3.863 & 0.002 & 1 & 10.904 & 0.033 \\
\hline Cerebrovascular disease & l & l & l & l & l & l & 18 & 0.105 & 0.044 \\
\hline [Average BC] & 3 & 1.154 & $<0.001$ & 5 & 1.103 & 0.005 & 5 & 1.302 & 0.010 \\
\hline Maximum BG & 8 & 1.116 & $<0.001$ & 7 & 1.085 & $<0.001$ & 7 & 1.182 & 0.007 \\
\hline Maximum SBP & 14 & 1.032 & $<0.001$ & 13 & 1.022 & 0.005 & 10 & 1.052 & 0.020 \\
\hline Maximum DBP & 13 & 1.037 & $<0.001$ & 11 & 1.024 & 0.044 & $l$ & l & l \\
\hline Total protein & 21 & 0.943 & $<0.001$ & 19 & 0.953 & 0.010 & 15 & 0.915 & 0.008 \\
\hline AST & 16 & 1.019 & 0.002 & 14 & 1.016 & 0.012 & $l$ & l & / \\
\hline Albumin & 22 & 0.904 & $<0.001$ & 20 & 0.922 & $<0.001$ & 16 & 0.816 & 0.060 \\
\hline BUN & 5 & 1.136 & $<0.001$ & 2 & 1.125 & 0.003 & l & l & / \\
\hline Creatine kinase & 19 & 1.003 & $<0.001$ & 17 & 1.002 & 0.013 & 11 & 1.028 & 0.015 \\
\hline Creatine kinase-MB & 12 & 1.049 & $<0.001$ & 10 & 1.032 & 0.021 & 9 & 1.168 & 0.007 \\
\hline LDH & 18 & 1.007 & $<0.001$ & 16 & 1.006 & $<0.001$ & 13 & 1.009 & 0.001 \\
\hline $\mathrm{CRP}$ & 17 & 1.014 & $<0.001$ & 15 & 1.011 & $<0.001$ & 11 & 1.028 & $<0.001$ \\
\hline White blood cell count & 9 & 1.097 & $<0.001$ & 9 & 1.069 & 0.005 & 3 & 1.416 & 0.009 \\
\hline Neutrophil percentage & 6 & 1.128 & $<0.001$ & 4 & 1.104 & $<0.001$ & 4 & 1.305 & 0.008 \\
\hline Lymphocyte percentage & 23 & 0.862 & $<0.001$ & 21 & 0.884 & $<0.001$ & 17 & 0.639 & 0.031 \\
\hline Neutrophil count & 7 & 1.121 & $<0.001$ & 6 & 1.092 & $<0.001$ & 2 & 1.501 & 0.001 \\
\hline Lymphocyte count & 24 & 0.069 & $<0.001$ & 22 & 0.134 & $<0.001$ & 19 & 0.002 & 0.003 \\
\hline Hemoglobin & 15 & 1.025 & 0.012 & 11 & 1.024 & 0.025 & $l$ & l & l \\
\hline Platelet count & 20 & 0.993 & $<0.001$ & 18 & 0.995 & 0.010 & 14 & 0.983 & 0.009 \\
\hline PT & 10 & 1.085 & $<0.001$ & 8 & 1.078 & $<0.001$ & $l$ & $l$ & l \\
\hline D-dimer & 4 & 1.143 & $<0.001$ & 3 & 1.123 & $<0.001$ & 6 & 1.186 & 0.007 \\
\hline
\end{tabular}

Abbreviation: BG, blood glucose; ALT, alanine aminotransferase; AST, aspartate aminotransferase; BUN, urea nitrogen; LDH, lactate dehydrogenase; CRP, C-reactive protein; PT, prothrombin time

The cut-off value of blood pressure related with adverse clinical outcomes in elderly hypertension COVID19 patients

The hypertension population were divided into 3 subgroups according to their blood pressure level. $40.5 \%$ had a maximum SBP of higher than $160 \mathrm{mmHg}$; and $49.4 \%$ had a maximum DBP of higher than 90mmHg. (Fig. 4A). 
Significant difference of clinical outcomes could be seen among different SBP subgroups in the hypertensive group. The difference of overall clinical outcomes was not statistically between the SBP (1) subgroup and the SBP (2) subgroup ( $P=0.412$ by the log-rank test), but was statistically between the SBP (1) subgroup and the SBP (3) subgroup ( $P=0.002$ by the log-rank test). There were $11.11 \%$ (at both 30 days and 60 days) cases with adverse clinical outcomes in SBP subgroup (1), 13.79\% (at 30 days) and $20.69 \%$ (at 60 days) cases in SBP subgroup (2), 53.12\% (at 30 days) and $71.87 \%$ (at 60 days) cases in SBP subgroup (3). At the same time, the difference of overall clinical outcomes between the DBP (1) subgroup and the DBP (2) subgroup ( $P=0.005$ by the log-rank test) or the DBP (3) subgroup $(P=0.02$ by the log-rank test) was statistically. There were $17.50 \%$ (at 30 days) and $22.50 \%$ (at 60 days) patients with adverse clinical outcomes in the DBP subgroup (1), 47.83\% (at 30 days) and $52.17 \%$ (at 60 days) patients in the DBP subgroup (2), $37.5 \%$ (at 30 days) and $62.5 \%$ (at 60 days) patients in the DBP subgroup (3). The difference among maximum SBP and DBP subgroups all increased sharply at about 5 days and continued over time, the increase was most pronounced at 30 days, and reached the platform at about 45 days (Fig. 4B).

The results of multivariate Cox-proportional hazard model analysis showed that maximum SBP (3), maximum DBP (2) and maximum BG were the independent risk factors of adverse clinical outcomes in hypertension population (Fig. 4C).

\section{Discussion}

Our study provides the clinical characteristics of elderly concerning the role of comorbidities in the setting of COVID-19. Similar to the previously reported results, hypertension, diabetes, and cardiovascular diseases are the most frequent comorbidities $(7,11,12)$. However, in our cohort, patients with hypertension reach $59.4 \%$ (79 of 133 ), $27.1 \%$ of total patients and $39.2 \%$ of the hypertension group have adverse clinical outcomes, which are higher than other studies(13). This might because the Huoshenshan Hospital prefers to treat severely ill patients and all enrolled cases are elderly in our study.

$\mathrm{K}-\mathrm{M}$ analyze, univariate and multivariate Cox hazard analysis in the total patients confirmed that hypertension is the most independent risk factor of developing adverse clinical outcome in elderly COVID19 patients, which consistent with previous research $(14,15)$. The pathophysiologic mechanisms are generally believed to be the following two aspects: a critical interaction between SARS-CoV-2 and the angiotensin-converting enzyme 2(ACE2) $(16,17)$; the hypotension medicines which act on ACE2 $(18,19)$. Our study shows that the percentage of good outcomes in hypertension decreases $50 \%$ and the risk of adverse outcomes increases more than 3 times. COVID-19 patients with hypertension should be given additional attention to prevent worsening of their clinical outcomes $(4,18)$. Instruction of blood pressure control in hypertensive patients is very important for clinical treatment of COVID-19.

We identify that among the many blood pressure indexes, the maximum systolic and diastolic blood pressure are independent risk factors. Further multivariate Cox-proportional hazard model indicates that maximum blood pressure $\geq 160 / 90 \mathrm{mmHg}$ is associated with increased adverse clinical outcomes in 
hypertension group. Former studies also suggest that low and stable BP are optimal to achieve a favorable prognosis for COVID-19 patients with coexisting hypertension(19-21), but no exact treatment target was put forward. Only a Spanish study showed that admission SBP $<120$ and $\geq 140 \mathrm{mmHg}$ was a predictor of higher all-cause mortality compared to SBP between $120-140 \mathrm{mmHg}(8)$. Our study shows a brand-new evidence for hypertension COVID-19 patients' treatment: it is no more befit to strictly control blood pressure lower than $160 / 90 \mathrm{mmHg}$ to the completely normal range.

The other highlight of our study is the treatment time point in COVID-19 patients with hypertension. The good outcomes in higher hypertension group of $\geq 160 / 90 \mathrm{mmHg}$ decrease sharply after the first week, then reached about $50 \%$ at one month, $50-70 \%$ at the second month, the effect of SBP plays more important role than DBP. Thus, we believe that early blood pressure control in elderly COVID-19 cases with hypertension was important for the clinical prognosis of disease.

Our research reveals several other prognostic factors in elderly COVID-19 patients with hypertension. (1) In addition to blood pressure, the most effective factor in the progression of COVID-19 hypertension patients in this cohort was maximum BG value, which was out of our expectation. This may be because the maximum BG value presents the worst pancreatic conditions and makes infection difficult to control $(11,22)$. (2) PT level was also an important risk factor for clinical outcome of elderly COVID-19 cases with hypertension, while $D$ - dimer is not mentioned in our study. Some studies suggest mild increases in PT are reminiscent of thrombotic microangiopathy(23-25). (3) CRP level was related to the adverse outcomes of COVID-19 patients, which associated with cytokine storms caused by virus invasion. In the systemic hyperinflammation phase of COVID-19, there is a significant elevation of inflammatory cytokines and biomarkers, which may lead to cardiopulmonary collapse and multi-organ failure $(26,27)$. (4) Hemoglobin level and platelet count were related to the adverse outcomes of COVID-19 patients. Thrombocytopenia was resulting from the consumption and/or the reduced production of platelets in damaged lungs. Anemia was not observed notably, but the decrease in hemoglobin was frequent $(28,29)$. (5) In this analysis, age was not included in the risk factors after adjustment for confounders, which might be because patients were all elderly, and the age span was relatively small.

Our study has the following limitations. First, the sample size of the study was small, especially in the non-hypertensive group. Second, due to the retrospective study design, the absence of ambulatory blood pressure monitoring results in all patients has limited the analysis of blood pressure control. Third, we did not have access to the data of patients before admission, so we were unable to evaluate the patient's previous blood pressure control. Fourth, patients admitted to Huoshenshan hospital were generally more serious, which made our study had an unavoidable selective bias.

\section{Conclusions}

In summary, hypertension is the most important independent risk factor of adverse outcomes in elderly COVID-19 patients, controlling the maximum blood pressure levels under 160/90 mmHg will decrease large part risks of adverse outcomes, the first week are key treatment period for patient prognosis. 


\section{Abbreviations}

COVID-19 = coronavirus disease 2019

$\mathrm{HR}=$ hazard ratio

$\mathrm{Cl}=$ confidence interval

SBP = systolic blood pressure

$\mathrm{DBP}=$ diastolic blood pressure

SARS-CoV-2 = severe acute respiratory syndrome coronavirus-2

ACE2 = angiotensin-converting enzyme 2

RAAS = renin-angiotensin-aldosterone system

$\mathrm{CT}=$ computed tomography

RT-PCR= reverse transcription-polymerase chain reaction

ICU = intensive care unit

$\mathrm{SD}=$ standard deviation

$\mathrm{IQR}=$ interquartile range

CRP $=$ C-reactive protein

BUN = blood urea nitrogen

$\mathrm{LDH}=$ lactate dehydrogenase

PT = prothrombin time

$B G=$ blood glucose

$\mathrm{ACE}=$ angiotensin-converting enzyme

ACEI = angiotensin-converting enzyme inhibitors

$\mathrm{ARB}=$ angiotensin receptor blockers

\section{Declarations}

1. Ethics approval and consent to participate 
Our study was conducted at the Huoshenshan Hospital in Wuhan, which was approved by the Institutional Ethics Committee of Huoshenshan Hospital (HSSLL030).

\section{Consent for publication}

Not applicable.

\section{Availability of data and materials}

The datasets used and/or analysed during the current study are available from the corresponding author on reasonable request.

4. Competing interests

Not applicable.

5. Funding

Not applicable.

\section{Authors' contributions}

$M W$ responded to the care of the patient. MW, LW, and $Y G$ were involved in the diagnosis, management, and treatment. MW and LW collected the data. TL, PZ and YW explained the data. YG, TL and CL designed the study. JZ and YW conducted the analysis. JZ and TL reviewed the relevant papers. YG, TL, and $\mathrm{CL}$ wrote the first draft. All authors contributed equally to writing the manuscript.

7. Acknowledgements

Not applicable.

\section{References}

1. Zhou P, Yang XL, Wang XG, Hu B, Zhang L, Zhang W, et al. A pneumonia outbreak associated with a new coronavirus of probable bat origin. Nature. 2020;579(7798):270-3.

2. Phelan AL, Katz R, Gostin LO. The Novel Coronavirus Originating in Wuhan, China: Challenges for Global Health Governance. Jama. 2020;323(8):709-10.

3. Worldometers. Coronavirus Update (Live) 2020 [

4. Kamyshnyi A, Krynytska I, Matskevych V, Marushchak M, Lushchak O. Arterial Hypertension as a Risk Comorbidity Associated with COVID-19 Pathology. Int J Hypertens. 2020;2020:8019360.

5. Xu H, Zhong L, Deng J, Peng J, Dan H, Zeng X, et al. High expression of ACE2 receptor of 2019-nCoV on the epithelial cells of oral mucosa. Int J Oral Sci. 2020;12(1):8. 
6. Rashedi J, Mahdavi Poor B, Asgharzadeh V, Pourostadi M, Samadi Kafil H, Vegari A, et al. Risk Factors for COVID-19. Infez Med. 2020;28(4):469-74.

7. Singh AK, Gillies CL, Singh R, Singh A, Chudasama Y, Coles B, et al. Prevalence of co-morbidities and their association with mortality in patients with COVID-19: A systematic review and meta-analysis. Diabetes Obes Metab. 2020;22(10):1915-24.

8. Rodilla E, Lopez-Carmona MD, Cortes X, Cobos-Palacios L, Canales S, Saez MC, et al. Impact of arterial stiffness on all-cause mortality in patients hospitalized with COVID-19 in Spain. Hypertension. 2020.

9. NHC C. New Coronavirus Pneumonia Prevention and Control Program (6th edition) 2020 [

10. Unger T, Borghi C, Charchar F, Khan NA, Poulter NR, Prabhakaran D, et al. 2020 International Society of Hypertension Global Hypertension Practice Guidelines. Hypertension. 2020;75(6):1334-57.

11. Zhang $P$, Wang $M$, Wang $Y$, Wang $Y, L i ~ T$, Zeng J, et al. Risk factors associated with the progression of COVID-19 in elderly diabetes patients. Diabetes Res Clin Pract. 2020;171:108550.

12. Meng M, Zhao Q, Kumar R, Bai C, Deng Y, Wan B. Impact of cardiovascular and metabolic diseases on the severity of COVID-19: a systematic review and meta-analysis. Aging (Albany NY). 2020;12(22):23409-21.

13. Lippi G, Wong J, Henry BM. Hypertension in patients with coronavirus disease 2019 (COVID-19): a pooled analysis. Pol Arch Intern Med. 2020;130(4):304-9.

14. Casucci G, Acanfora D, Incalzi RA. The Cross-Talk between Age, Hypertension and Inflammation in COVID-19 Patients: Therapeutic Targets. Drugs Aging. 2020;37(11):779-85.

15. Posso M, Comas M, Román M, Domingo L, Louro J, González C, et al. Comorbidities and Mortality in Patients With COVID-19 Aged 60 Years and Older in a University Hospital in Spain. Arch Bronconeumol. 2020;56(11):756-8.

16. Bansal R, Gubbi S, Muniyappa R. Metabolic Syndrome and COVID 19: Endocrine-Immune-Vascular Interactions Shapes Clinical Course. Endocrinology. 2020;161(10).

17. Devaux CA, Rolain JM, Raoult D. ACE2 receptor polymorphism: Susceptibility to SARS-CoV-2, hypertension, multi-organ failure, and COVID-19 disease outcome. J Microbiol Immunol Infect. 2020;53(3):425-35.

18. Huang S, Wang J, Liu F, Liu J, Cao G, Yang C, et al. COVID-19 patients with hypertension have more severe disease: a multicenter retrospective observational study. Hypertens Res. 2020;43(8):824-31.

19. Ran J, Song Y, Zhuang Z, Han L, Zhao S, Cao P, et al. Blood pressure control and adverse outcomes of COVID-19 infection in patients with concomitant hypertension in Wuhan, China. Hypertens Res. 2020;43(11):1267-76.

20. Chen R, Yang J, Gao X, Ding X, Yang Y, Shen Y, et al. Influence of blood pressure control and application of renin-angiotensin-aldosterone system inhibitors on the outcomes in COVID-19 patients with hypertension. J Clin Hypertens (Greenwich). 2020;22(11):1974-83. 
21. Caillon A, Zhao K, Klein KO, Greenwood C, Lu Z, Paradis P, et al. High systolic blood pressure at hospital admission is an important risk factor in models predicting outcome of COVID-19 patients. Am J Hypertens. 2021.

22. Wang $H$, Tang $X$, Fan $H$, Luo $Y$, Song $Y, X u Y$, et al. Potential mechanisms of hemorrhagic stroke in elderly COVID-19 patients. Aging (Albany NY). 2020;12(11):10022-34.

23. Iba T, Levy JH, Levi M, Connors JM, Thachil J. Coagulopathy of Coronavirus Disease 2019. Crit Care Med. 2020;48(9):1358-64.

24. Iba T, Levy JH, Levi M, Thachil J. Coagulopathy in COVID-19. J Thromb Haemost. 2020;18(9):2103-9.

25. Iba T, Levy JH, Connors JM, Warkentin TE, Thachil J, Levi M. The unique characteristics of COVID-19 coagulopathy. Crit Care. 2020;24(1):360.

26. Huang I, Pranata R, Lim MA, Oehadian A, Alisjahbana B. C-reactive protein, procalcitonin, D-dimer, and ferritin in severe coronavirus disease-2019: a meta-analysis. Ther Adv Respir Dis. 2020;14:1753466620937175.

27. Sahu BR, Kampa RK, Padhi A, Panda AK. C-reactive protein: A promising biomarker for poor prognosis in COVID-19 infection. Clin Chim Acta. 2020;509:91-4.

28. Liu X, Zhang R, He G. Hematological findings in coronavirus disease 2019: indications of progression of disease. Ann Hematol. 2020;99(7):1421-8.

29. Mansueto G, Niola M, Napoli C. Can COVID 2019 induce a specific cardiovascular damage or it exacerbates pre-existing cardiovascular diseases? Pathol Res Pract. 2020;216(9):153086.

\section{Figures}

\section{0 patients diagnosed with COVID-19 between Feb 8, 2020 to Mar 17, 2020}

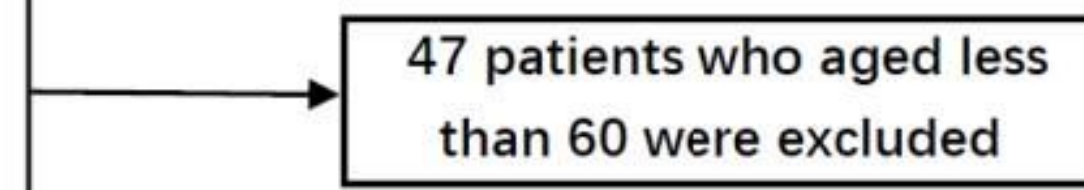

\section{COVID-19 patients included in the study}

Hypertension patients

$$
n=79
$$

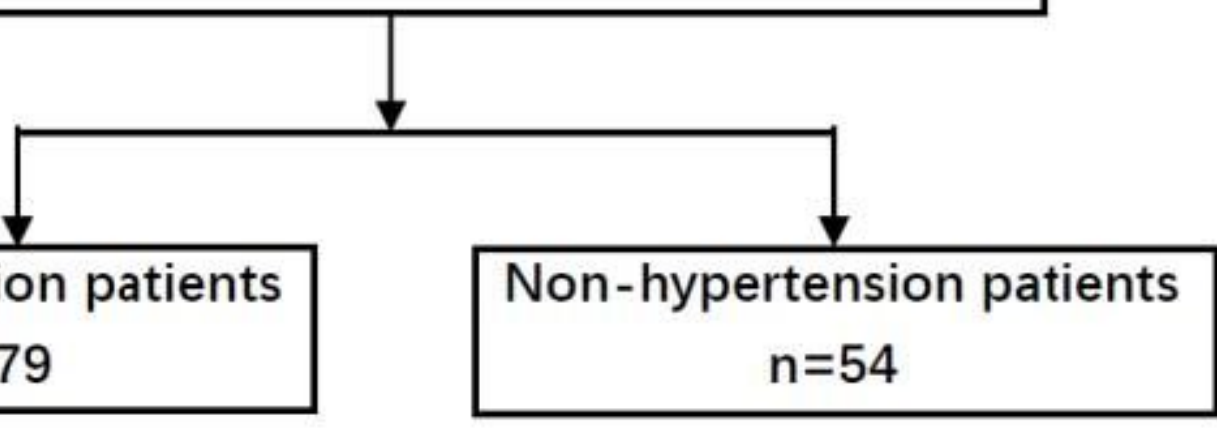


Figure 1

Diagram of study inclusion criteria

A

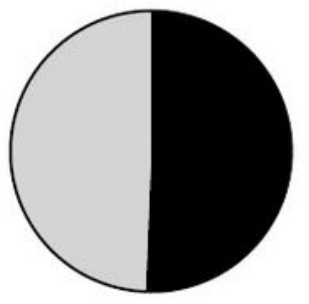

Hypertension(n=79)
- $50.63 \%$ Severe $(n=40)$ $\square 49.37 \%$ Mild to moderate $(n=39)$

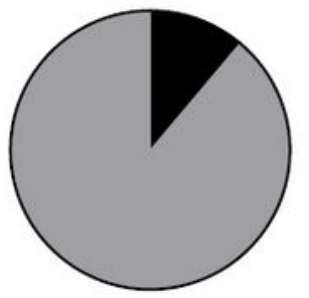

Non-hypertension( $n=54)$

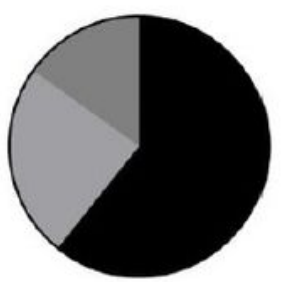

Hypertension $(n=79)$

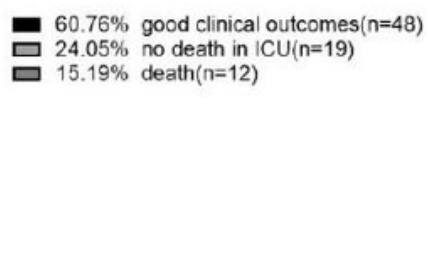

Non-hypertension( $n=54)$

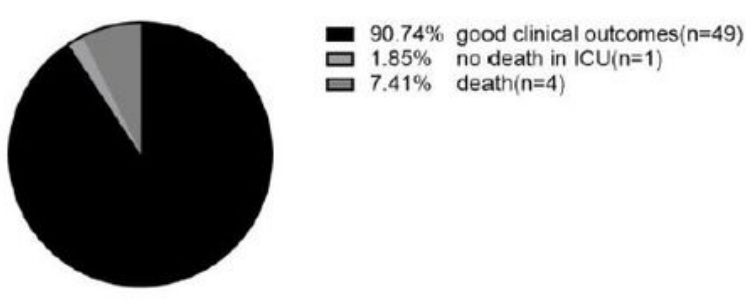

$11.11 \%$ Severe $(n=6)$

$88.89 \%$ Mild to moderate $(n=48)$

b $\square 1.85 \%$ no death in $I C U(n=1)$ $7.41 \%$ death $(n=4)$

B

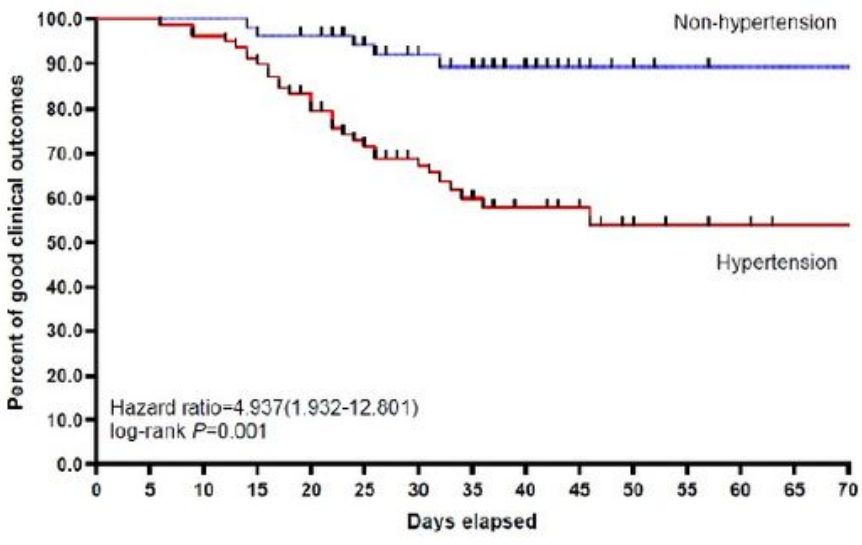

\section{No.at risk}

$\begin{array}{lllllllll}\text { Hypertension } & 79 & 76 & 63 & 53 & 50 & 48 & 48 & 48 \\ \text { Non-hypertension } & 54 & 54 & 52 & 50 & 49 & 49 & 49 & 49\end{array}$

\section{Figure 2}

The difference of adverse clinical outcomes between hypertension and non-hypertension patients. A a: clinical classification in hypertension, b: clinical classification in non-hypertension, c: clinical outcomes in 
hypertension, d: clinical outcomes in non-hypertension B: Kaplan-Meier Analysis of the precent of good clinical outcomes between hypertension and non-hypertension cases. Abbreviation: ICU, intensive care unit

Risk Factors Hazard Ratio $(95 \% \mathrm{Cl}) \quad P$ value

\begin{tabular}{rrrr}
\hline Hypertension & $3.202(1.164,8.807)$ & 0.024 \\
LDH & $1.006(1.004,1.009)$ & $<0.001$ \\
CRP & $1.008(1.003,1.012)$ & 0.001 \\
Lymphocyte count & $0.166(0.056,0.494)$ & 0.001 \\
Hemoglobin & $1.030(1.012,1.048)$ & 0.001 & \\
Platelet count & $1.004(1.000,1.009)$ & 0.042 & 0.0
\end{tabular}

\section{Figure 3}

The multivariate Cox-proportional hazard model analysis to further certify whether variables affected patient clinical outcomes in the total population 


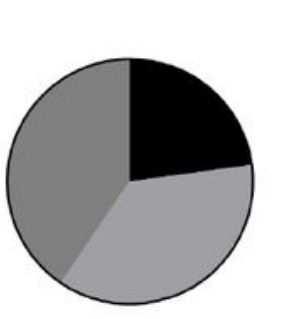

Hypertension( $n=79$ )

a

B

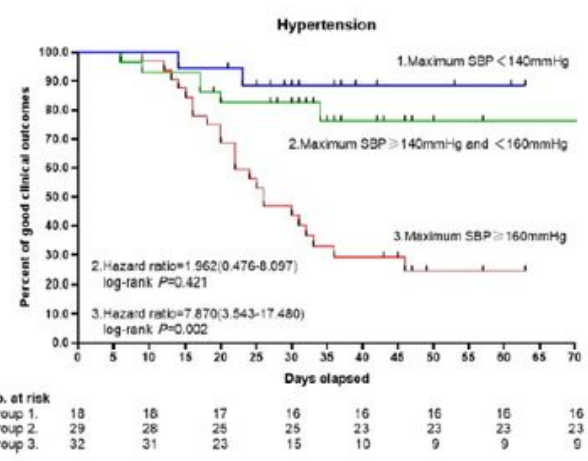

a
Maximum SBP

- $22.8 \%<140 \mathrm{mmHg}(\mathrm{n}=18)$

$36.7 \% \quad 140-159 \mathrm{mmHg}(\mathrm{n}=29)$

$40.5 \% \geqslant 160 \mathrm{mmHg}(\mathrm{n}=32)$

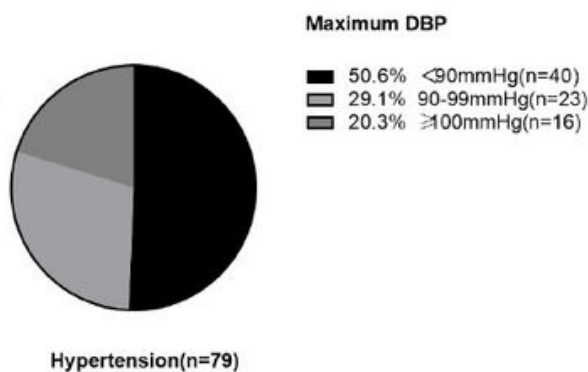

b

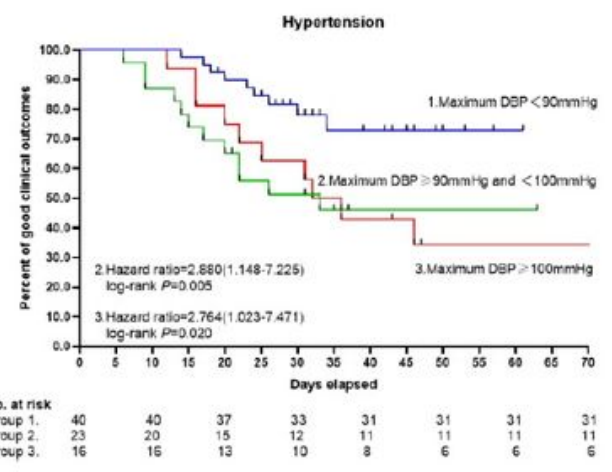

b

C

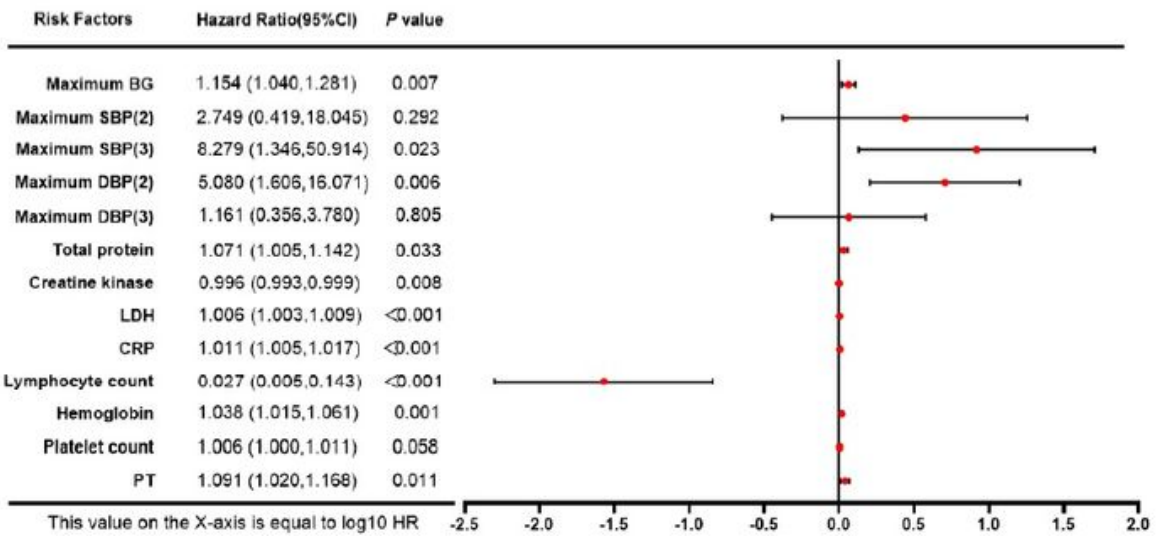

Figure 4

The cut-off value of blood pressure related with adverse clinical outcomes in elderly hypertension COVID19 patients. A a: the hypertension population were divided into 3 subgroups according to their maximum SBP, b: the hypertension population were divided into 3 subgroups according to their maximum DBP. B Kaplan-Meier Analysis of the precent of good clinical outcomes between different blood pressure subgroups. a: Maximum SBP in the hypertension group, b: Maximum DBP in the hypertension group. C 
The multivariate Cox-proportional hazard model analysis to further certify whether variables affected patient clinical outcomes in the hypertension group 